\title{
THEORETICAL CONSIDERATIONS RELATING TO THE SINGLE-LINED AND THE MANY-LINED SPECTRUM OF MERCURY.
}

\section{R. A. Millikan.}

$A^{\mathrm{T}}$ the meeting of the Physical Society of Chicago in November, A I9I4, I raised a question as to the reality of the so-called one-line spectrum which had seemed to appear in Franck's and Hertz's experiment. I pointed out that if we took the point of view of the pure experimentalist, Wood's demonstration of the enormous absorbing and emitting power of mercury vapor for line of wave-length $2536 \AA$. along with its transparency for other lines means that with increasing volume excitation the relative intensity of these other lines to that of 2536 must continually increase. Otherwise stated, the opacity of the mercury vapor for line 2536 requires that the light of this wave-length which comes out of a tube come only from the surface layers, while the light of other wave-lengths will come from the whole volume. With weak excitation, therefore, line 2536 might be enormously more intense than the other lines and thus appear on Franck's and Hertz's photographic plates, although the other lines might still be present but merely be too weak to be noticed with the exposures used.

On the other hand, from the point of view of Bohr's theory, a singleline spectrum is not to be expected at least at line 2536 . This can be seen from the following considerations: It has long been suspected, and indeed was proved more than a year ago by Tait $^{1}$ and again quite recently by Goucher, ${ }^{2}$ that though when negative electrons are driven through mercury vapor there is inelastic impact at 4.9 volts, there is very little ionization at that point or indeed at any potential less than about I0.4 volts when strong ionization sets in. This means from the point of view of Bohr's theory, that at 4.9 volts an electron is merely displaced from one particular orbit to the next outer orbit and when it returns it emits the longest line of the series corresponding to a return to this particular orbit. Line 2536 should therefore be the longest wavelength in a series, as it is in fact, and none of the shorter wave-lengths

1 Phys. Rev., VII., 686, r9i6.

2 Phys. Rev., VIII., 56I, I9r6. 
of this particular series should appear at 4.9 volts, but the whole series should appear when this electron is knocked entirely out of the atom. This would occur, let us assume, at I0.4 volts, a potential which corresponds, in accordance with the quantum relation, to the convergence wave-length (II $88 \AA$.) of the series of which line 2536 is the long wavelength head. The many-lined spectrum which should suddenly appear at I0.4 volts should then be merely the whole series of which line 2536 is the member of longest wave-length.

But now, since the normal spectrum of mercury actually has many lines of greater wave-length than 2536, these must, according to the Bohr theory, belong to orbits farther out from the nucleus than that producing line 2536 for its member of longest wave-length, and these lines should always be produced when 2536 is produced. For if in the permanent state of mercury vapor some of its atoms have electrons in these outer orbits, they must be knocked entirely out at potentials which should not be greater and may be much less than 5.5 volts. To see how this follows from the Bohr theory let the subscripts I and 2 apply to any two adjacent series, such as the $\mathrm{K}$ and $\mathrm{L}$ series in $\mathrm{X}$-rays. The Bohr theory then states that

$$
\nu_{\beta_{1}}-\nu_{\alpha_{1}}=\nu_{\alpha_{2}} .
$$

Kosse $^{1}$ first published tests of this relation taken from X-ray data. The equation gives also the correct relation between the Lyman ultraviolet series of hydrogen, the Balmer series, and the Paschen infra-red series, since these all fit perfectly the Bohr theory. Even where the simple form of Bohr's theory, which it will be recalled was developed only for a single negative electron rotating about a positive center, does not apply, the foregoing equation should still hold, for it states merely the energy relations which must be satisfied no matter what influences the electrons in the various orbits may exert upon one another. ${ }^{2}$ If then, $\nu_{\infty_{1}}$ and $\nu_{\infty_{2}}$ represent the frequency limits toward which the series corresponding to two adjacent orbits run, then the energy relations underlying equation I may also take the form

$$
\nu_{\infty_{1}}-\nu_{a_{1}}=\nu_{\infty_{2}} .
$$

This is merely the well-known Rydberg-Schuster law ${ }^{3}$ discovered wholly empirically and tested quite generally for metallic spectra, but now seen to be a necessary consequence of the Bohr theory of the atom.

1 Verh. d. D. Phys. Ges., 16, 953, r9r4.

2 See Presidential Address American Physical Society on "Radiation and Atomic Structure," Phys. Rev., May, I9I7.

3 Bailey's Spectroscopy, p. 488. 
Now the series of which the mercury line 2536 is the long wave-length head runs toward the limit $\lambda=$ I $88 \AA$. Applying equation 2, we obtain

$$
\frac{\mathrm{I}}{\mathrm{II} 88}-\frac{\mathrm{I}}{2536.7}=\frac{\mathrm{I}}{x} \therefore x=2234 \text {. }
$$

This is indeed exactly the short wave-length limit toward which one of the well-known mercury series runs, viz., the series $3 \mathrm{I} 25,265^{2}, 2482, \cdots$, 2234, and according to the Bohr theory it should be the series which corresponds to the orbit just outside that which gives rise to the series of which line 2536 is the member of longest wave-length. The value of $h \nu$ in volts corresponding to this wave-length 2234 , is 5.5 . If, then, there are electrons in this orbit they should be knocked entirely out at 5.5 volts and the whole series of which line 2234 is the convergence frequency should appear. The longer wave-length members of this series should appear at still lower potentials; if they do not so appear it should be because there are no electrons in these orbits.

But even if in the normal mercury vapor there are no electrons in orbits which are larger than that corresponding to the series whose longest wave-length member is 2536 , yet since this line is stimulated it must act photoelectrically, as Van der Bijl1 has just been urging, on the surrounding atoms, for the photoelectric long wave-length limit of mercury is about $2800 \AA$. and the return of these detached electrons, or of others which take their places, should be accompanied by all lines of series corresponding to outer orbits which, according to our hypothesis, are normally empty. As a matter of fact the lines which do appear faintly but altogether definitely on both I. and III. of the foregoing plate, though less clearly on the reproduction, are 3125 and 2652 of the above-mentioned series and in addition 2967 and 3022, which, as Prof. F. A. Saunders kindly tells me, are members of the same triplet series; this series being

$\begin{array}{lllll}3650 & 3022 & 2804 & \ldots & 249 \mathrm{I} \\ 3 \mathrm{I} 25 & 2652 & 2482 & \ldots & 2234 \\ 2967 & 2534 & 2378 & \ldots & 2 \mathrm{I} 49 .\end{array}$

In the preceding discussion it has been assumed that the emission of light by mercury is due solely to the partial or complete formation of positive atom-ions. But it is conceivable that the light-emission is in part at least due to the simple stimulation of neutral mercury atoms by corpuscular impact. In this case many lines would be expected to appear simultaneously and at a potential which could scarcely be expected to bear any relation to the value of $h \nu$ for any one of the lines.

${ }^{1}$ Proc. Phys. Soc., Chicago meeting, Dec. I, Igr6. 
From whatever point of view, then, I approach the excitation of mercury vapor I can see no reason to expect line 2536 to appear without the lines of longer wave-length. These lines would be expected to be weak if there are few or no electrons in orbits greater than that corresponding to 2536 , but they should nevertheless be present. I initiated Dr. Hebb's experiments, therefore, in the hope that they would bring to light these weak lines of longer wave-length than 2536 and accompanying it.

His results seem to me to indicate strongly that these lines are in fact always present along with 2536 , their appearance on the photographic plate depending, as is to be expected from Wood's experiments, simply on the volume excitation, or in a given tube, upon the density of the exciting electron stream. Thus in I. of the plate the reason these additional lines appear in the upper half and not in the lower is that, as is well known, the electron stream is a sharply limited bundle outside of which the excitation is too weak to permit the appearance of the weaker lines in the exposure time used. The sharp limits of the cathode beam explain fully the bluntness of the ends, without assuming that a definite density is necessary to stimulate the many-lined spectrum. That these weak lines are present in II. when the P. D. is 20 volts but the electron stream weak, no one, I fancy, would be inclined to doubt.

The experiments on the starting potential of the arc seem to me to demonstrate, first, that this potential depends upon the density of the exciting cathode stream; second, that this potential may be anything above five volts.

Both of these conclusions are in accordance with the foregoing theory. For the striking of an arc merely indicates the rise in the number of positive ions to a certain large value and the consequent concentration of the potential-fall in the neighborhood of the cathode. But with the reciprocal relations which have been postulated between the stimulation of light at 4.9 volts and the photoelectric production of ions by this light, there may exist at any potential in excess of 4.9 volts, the possibility of an indefinite multiplication of positive ions. With a weak cathode stream this possibility will not be realized in the neighborhood of 5 volts, since, in view of the strong absorption by mercury vapor of line 2536 the photoelectrons will be formed close to where the light is stimulated, $i$. e., close to the anode. The negatives so formed will not then fall through an appreciable potential and hence will not help the original cathode stream in the stimulation of light. The denser the original cathode stream, however, the more positive ions will be formed by the light and consequently the more will the total fall of potential become 
concentrated near the cathode, and the sooner will negative photoelectric ions begin to be formed where they can aid the original cathode stream in the stimulation of more light. As soon as this condition ensues there arises the possibility of an indefinite multiplication of positive ions and the formation of an arc.

If the cathode stream is weak then the arc will not appear in the neighborhood of 5 volts. It would, however, be expected to appear at the ionizing potential, namely, at I0.4 volts, since at this point there is in any case a large multiplication of ions. This is where the arc actually did appear in McLennan and Henderson's experiments. ${ }^{1}$

It may be mentioned that the fact that no measurable ionization has ever been observed in mercury vapor at potentials lower than 4.9 volts would signify, from the point of view here presented, that when the mercury atom is in its normal, stable condition, the orbit corresponding to line 2536 is the outermost one which contains any appreciable number of its 80 negative electrons.

Whether the foregoing theory is correct or not there can be no doubt that a mercury arc can be maintained at much less than I0.4 volts, for I have myself held one at 3.5 volts, but in this case as in Dr. Hebb's experiments, I suspect that the contact electromotive force was sufficient to raise the actual energy of impact to 4.9 volts. As further evidence it may be mentioned that $\mathrm{Rau}^{2}$ observed the appearance of the mercury line 4359 at 8 volts, which is below the value, I0.4 volts, which McLennan found to be the lowest at which any of the lines of the many-lined spectrum could appear.

That the quantum relation holds both at 4.9 volts and at the ionization potential, I0.4, volts is rendered probable by Tait's and by Goucher's experiments, as well as by those of McLennan; for both the observed potential of inelastic impact, and that of ionization seem to agree, within the limits of error of the experiments, with predicted values. The present experiments are not at all at variance with McLennan's but they extend them by showing, first, that an arc may be produced without raising the potential to the ionizing value, and second, that weak lines of longer wave-length than 2536 probably always appear with it. It is only the shorter and shorter wave-lengths of a given series which ought to appear successively with successive increases in the applied potential. This prediction is also in line with Rau's results.

Ryerson Physical LABORATORY, University of Chicago.

1 McLennan and Henderson, Proc. Roy. Soc., 9I, 485, I9I5, and McLennan and Keys, Proc. Roy. Soc., 92, 59I, I9I6.

${ }^{2}$ H. Rau, Ber. d. Phys. Med. Ges. Wurzburg, I9I4. 